\title{
Life at the poles
}

Despite its harsh climate, the Arctic Circle is home to a rich fauna, including the male ptarmigan pictured here. A yearround resident of the Arctic, this bird retains his summer plumage on his head, with white winter feathers on his body. In A Complete Guide to Arctic Wildlife (A\&C Black, E40), Richard Sale provides an extensive field guide to the birds and mammals that inhabit this harsh and unforgiving environment. The book also provides an introduction to the geology and geography of the region, shows how this has shaped the organisms that live there, and

discusses the impact of humans on this fragile environment.

The World of the Polar Bear (Firefly, $£ 29.99$, \$45) features a collection of stunning photographs by Norbert Rosing. The book explores a year in the life of the polar bear and the environment it inhabits.

Surviving Antarctica by David N. Thomas, to be published by Natural History Museum Publishing next month ( $E 9.99$ ), examines how endemic inhabitants and human visitors at the other pole survive in one of the most inhospitable regions on the planet.

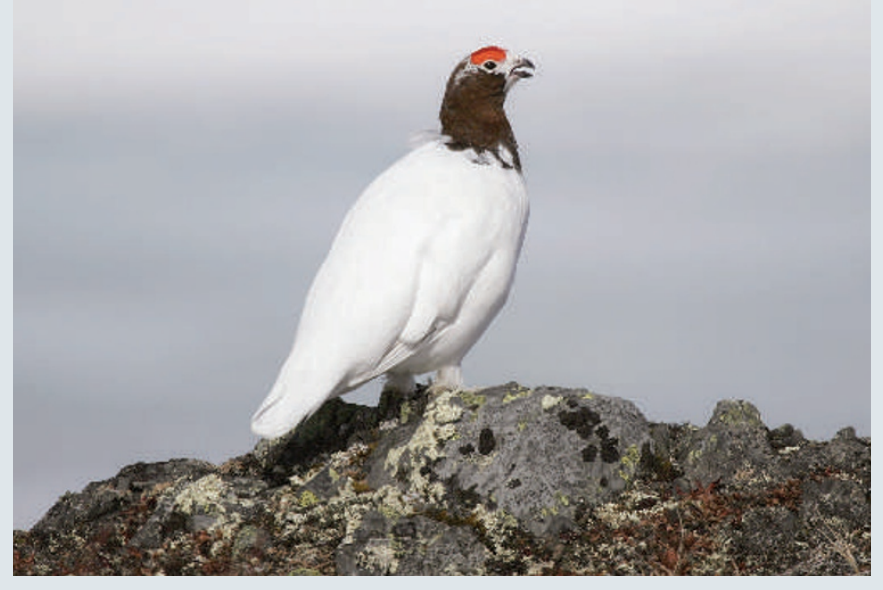

\section{The changing view of autism}

\section{Unstrange Minds: Remapping the World of Autism \\ by Roy Richard Grinker \\ Basic Books: 2007. 304 pp. \$26}

\section{Lorna Wing}

The term 'autism' was coined by Leo Kanner in 1943 as a label for a disordered pattern of behaviour present from infancy that he and his colleague Leon Eisenberg had observed. They defined it very narrowly, as a profound lack of affective contact with other people, combined with an obsessive desire for the preservation of 'sameness' that results in elaborate, repetitive routines.

In Unstrange Minds, Roy Grinker combines his experiences as an anthropologist and as a father of a teenage daughter with autism, Isabel, to provide a fresh view of the challenges posed by this condition. He interweaves three themes: first, the evolution of ideas about the nature of autism and the effect of these changes on studies of prevalence; second, the intimate connection between cultural attitudes to autism and the quality of life for those affected and their families; and third, the story of his own daughter's development and the struggles that he and his wife have experienced in the United States to ensure that Isabel has the support and education she needs.

Grinker's discussion of the changing concepts of autism and its prevalence is highly topical. Some people believe that the number of children with autism has increased at an alarming rate since the 1990s and describe this as an 'autism epidemic'. But many others think that most, if not all, of the rise in prevalence reported in epidemiological studies can be explained by increasing awareness and a widening of diagnostic criteria. Feelings run high, especially among those who believe that, despite the lack of convincing scientific evidence, the increase is due to environmental causes such as pollution or a combined vaccination against mumps, measles and rubella (the MMR vaccine).

Grinker firmly rejects this view and rebuts any suggestion of an autism epidemic. He gives a compelling account of the complex way in which our understanding of the nature of autistic conditions has evolved. Children and adults with autistic behaviour have always existed, but in the past they were considered to be suffering from other conditions such as mental retardation, behaviour disorder or, in some cultures, some sort of malign influence. Once autism was identified as a childhood condition, researchers, clinicians and parents all contributed to the changes in diagnostic criteria.

Autism is no longer seen simply as Kanner's precisely defined, very rare condition, affecting as few as one or two children in every 10,000 and separate from all other childhood disorders. It is viewed instead as part of a spectrum of disorders of brain development causing impairments in social interaction, social communication and social imagination, along with a limited, repetitive pattern of behaviour. This spectrum has very wide manifestations that overlap with other developmental disorders and occur in people of any level of ability. The best evidence suggests a prevalence of around 10 in 1,000, even higher than the 6 in 1,000 quoted by the author. Grinker sees the higher reported prevalence as good news, as it indicates growing understanding and acceptance.

Grinker's exploration of cultural differences in attitudes to autism is very moving. He describes the experiences of individual children and their families in South Africa (a Zulu family), the United States, India and
South Korea. Each culture has very different explanations for the strange behaviour of these children. However, in all societies, the main burden of caring for a child with autism is borne by the mother. During much of the twentieth century, especially in the West, the distress they suffered was intensified by psychiatrists and psychoanalysts who blamed the mother for the child's strange behaviour. The fact that there are no visible signs of the brain disorder underlying autism reinforced the tendency to look for a non-physical reason for the socially unacceptable behaviour. In some other societies, the shame of having a child with a developmental disorder adds immeasurably to the family's problems. One of the messages of this book is that personal experience of any illness or disability is deeply affected by the culture in which one lives.

Isabel's story is interwoven with the other themes. Her parents' acceptance and love for her is evident in everything Grinker writes. She has typically autistic visuospatial and memory skills, and a high IQ if the measurement is based on these abilities. But this is combined with the autistic lack of inborn social skills, so she has trouble adapting her abilities to social demands. It is strange to think how recently psychologists became aware that social skills are an important dimension of overall ability.

As a mother of a daughter with autism, I know that parents will empathize with the author and be fascinated by his view of the subject and the stories he tells. As a professional, I recommend this book to those working in the field for its thought-provoking examination of important themes.

Lorna Wing is honorary consultant psychiatrist at the Centre for Social and Communication Disorders, 113 Masons Hill, Bromley,

Kent BR2 9HT, UK. 